Case Report

\title{
Improved Awareness of Tuberculosis Infection in Advanced Stage Chronic Renal Disease Could Reduce Cases of Active TB: Lessons from Four Challenging Cases
}

Siva Mahendran ${ }^{1,}{ }^{*}$, Paramit Chowdhury ${ }^{2}$, Ronan Breen ${ }^{3}$, Grainne d'Ancona ${ }^{3,4}$, Heather J. Milburn 3,4

1. Consultant Respiratory Physician Kingston Hospital, Galsworthy Road, Kingston Upon Thames KT2 7QB; E-Mail: sivamahendran@nhs.net

2. Department of Renal Medicine, Guy's and St Thomas' Hospital, Great Maze Pond, London SE1 9RT; E-Mail: paramit.chowdhury@kcl.ac.uk

3. Department of Respiratory Medicine, Chest Clinic, Guy's and St Thomas' Hospital, Great Maze Pond, London SE1 9RT; E-Mails: Ronan.breen@gstt.nhs.uk; Grainne.dAncona@gstt.nhs.uk; heather.j.milburn@kcl.ac.uk

4. King's College London, Great Maze Pond, London SE1 9RT.

* Correspondence: Siva Mahendran; E-Mail: sivamahendran@nhs.net

Academic Editor: Wasim A. Dar

Special Issue: Infectious Complications in Kidney Transplantation

OBM Transplantation

2021, volume 5 , issue 1

doi:10.21926/obm.transplant.2101137
Received: August 14, 2020

Accepted: February 21, 2021

Published: March 08, 2021

\section{Abstract}

Patients with chronic kidney disease (CKD) have an increased risk of developing tuberculosis (TB) compared to those with normal renal function. The reasons for this are well described, but include impaired cellular immunity, a high incidence of co-morbid conditions as well as the concomitant use of immunosuppressive medications. Ethnicity as well as socio-economic factors also prevail. Expert guidelines recommend TB chemoprophylaxis in renal transplant recipients deemed at high risk - invariably those from ethnic minorities or recent arrivals from areas with high endemic rates of TB. However, in most renal centres within the UK including our own, high risk patients with advanced CKD (stages 4 and 5) are not routinely screened for

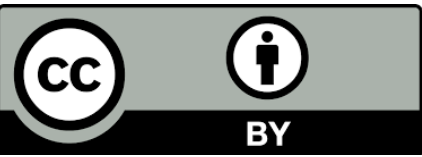

(C) 2021 by the author. This is an open access article distributed under the conditions of the Creative Commons by Attribution License, which permits unrestricted use, distribution, and reproduction in any medium or format, provided the original work is correctly cited. 
latent TB infection (LTBI) prior to transplantation, thus contributing to missed opportunities for preventing TB disease. We report four challenging cases of patients diagnosed with TB disease on a background of advanced CKD, who all presented within an 18-month period whilst under the care of a large tertiary renal centre. Two of the cases were patients with wellfunctioning renal transplants and two were on renal replacement therapy. We describe our experience and share practical considerations on how to manage TB in CKD including the role of therapeutic drug monitoring in peritoneal dialysis. These cases highlight the need for a better awareness of the possibility of TB reactivation and underscore the arguments in favour of screening programmes for LTBI in patients with advanced CKD.

\section{Keywords}

Tuberculosis; peritoneal dialysis; chronic kidney disease; renal transplantation; therapeutic drug monitoring

\section{Introduction}

Patients with advanced chronic kidney disease (CKD) have an increased risk of developing Mycobacterium tuberculosis (TB) infection and disease compared with those with normal renal function [1]. The main reasons for this are impaired cellular immunity, immunosuppressive medications, diabetes mellitus and co-infection with human immunodeficiency virus (HIV). Patients from ethnic minorities are at particular risk of developing both TB and CKD [2]. Socio-economic and lifestyle factors also contribute [3]. In patients with CKD, the diagnosis of TB can be difficult and delayed due to non-specific symptoms and a higher incidence of extra-pulmonary involvement. Within renal transplant recipients, TB infection and disease is associated with increased mortality and allograft loss $[4,5]$.

Estimates of the relative risk (RR) of developing TB infection and disease amongst patients with CKD are varied and confounded by some of the factors listed above. The British Thoracic Society (BTS) guidelines on the clinical management of TB in patients with CKD [6] cited a 10-25-fold higher risk of developing TB amongst haemodialysis (HD) patients, when compared with the general population. In a recently published study in London, when compared with background TB rates, the risk of developing TB infection and disease was 29-fold, 9-fold and 12-fold higher in HD, PD and transplant recipients respectively [7]. In areas endemic for TB, the RR is also higher, although at a lower adjusted risk at between 2-4 times background rates [1, 8].

In renal transplant recipients, the incidence of TB infection is mainly determined by the prevailing epidemiological risk in the country with estimates that range from $0.34 \%$ in Spain - a low TB burden country [9] to $15 \%$ in Pakistan - a high TB burden country [10].

Set against this, the BTS and other expert groups, recommend TB chemoprophylaxis in all graft recipients of Indo-Asian or African heritage as well as anyone who has been living in the UK for under 5 years and is from a country with TB incidence rates above 40/100,000 [6]. They also advocate individual risk assessments in those with CKD and on transplant waiting lists for those at risk of TB infection. However, most renal centres in the UK including our own, do not routinely screen patients for latent TB infection (LTBI), but give routine isoniazid (with pyridoxine) 
chemoprophylaxis to all post-transplant patients. This can result in over treatment of some patients but also significant drug interactions with concomitant immunosuppression [11].

We present four cases of TB infection in advanced CKD where a better awareness of the possibility of TB reactivation might have reduced the chances of subsequent development of active disease. All four patients presented over an 18-month period between 2014 - 16, whilst under the care of a large tertiary renal service. In two of the patients, a diagnosis other than TB infection was originally thought to be the most likely. All four patients were successfully treated but their care raised challenging treatment decisions in the context of renal transplant or renal replacement therapy (RRT).

\section{Case 1}

A 40 year-old female of Kenyan origin was admitted for cadaveric renal transplantation. She had CKD secondary to focal sclerosing glomerular sclerosis (FSGS) which had been managed with continuous ambulatory peritoneal dialysis (CAPD) for the previous six months.

Upon admission, initial assessment and routine blood tests were satisfactory. She received standard pre-transplant induction therapy using Basiliximab (an IL-2 receptor inhibitor) and methylprednisolone. During exploration of her abdominal cavity, two $3 \mathrm{~cm}$ abnormal necrotic lymph nodes (LN) were found within the pelvis for which frozen section revealed necrotizing granulomatous disease and her transplant was abandoned. Ziehl-Neelsen (ZN) staining of the LNs confirmed the presence of acid-fast bacilli (AFB) and polymerase chain reaction (PCR) confirmed $M$. tuberculosis. Staging CT revealed necrotic mediastinal and pelvic lymph nodes but no other sites of disease.

On further review, the patient gave a 2-week history of drenching night sweats and weight loss. The patient's mother had been treated for TB when the patient was a child living in Kenya. However, her mother had remained in Kenya and the patient had not returned to Kenya for at least 5 years and had no other known TB contacts. The patient was started on daily rifampicin (R), isoniazid $(H)$, moxifloxacin and pyridoxine together with three times weekly pyrazinamide $(Z)$. The patient was suspended from the transplant list and continued on daily peritoneal exchanges. Mycobacterial culture identified a fully sensitive organism and, following two months of treatment she was stepped down to daily $\mathrm{RH}$ in line with standard care.

Therapeutic drug monitoring (TDM) of $\mathrm{R}$ and $\mathrm{H}$ identified undetectable trough drug levels, and low post-dose levels measured at 2 and 4 hours, leading to increased doses of $R$ and $H$. Meanwhile, trough and post dose levels for $Z$ were high, necessitating a dose reduction. TDM proved challenging due to the time at which the patient usually took her medications and subsequent measurement of timed drug levels within the out-patient setting. Her continuation phase of treatment was lengthened to six months in view of the low post-dose drug levels and to mitigate against the possibility of sub-optimal treatment. She made a complete recovery and has since been successfully transplanted.

\section{Case 2}

A 51 year-old UK-born female of Nigerian ancestry and a cadaveric donor recipient was admitted with low-grade fevers, night sweats and abdominal pain. Two years previously, she had been transplanted for FSGS, and prior to that, had required RRT with CAPD. Her transplantation had been 
uncomplicated and, in line with guidelines, she had been commenced on isoniazid TB chemoprophylaxis. Eight days into TB chemoprophylaxis, her alanine transaminase (ALT) levels increased to an equivalent of four times the upper limit of normal (ULN). Isoniazid was withdrawn and her ALT level normalised, but further TB chemoprophylaxis was not offered. She had then remained well, with good graft function and stable immunosuppression on tacrolimus, mycophenolate mofetil and prednisolone.

On assessment, her abdomen was noted to be mildly distended but otherwise soft on examination. CT identified a normally enhancing renal transplant but mild peritoneal thickening and small volume ascites. Abdominal paracentesis was performed for which fluid analysis showed a raised WCC count at 740 cells $/ \mathrm{mm}^{3}$ with mainly reactive lymphocytes and neutrophils. ZN stains for AFBs were negative as was routine bacterial culture and tests for Epstein-Barr virus (EBV). Immunocytochemistry analysis found no evidence of a lymphoproliferative disorder.

An explorative laparoscopy identified caseous fibrinous adhesions throughout (Figures 1a, 1b). Peritoneal biopsy revealed granulomatous infection with a single focus of necrosis consistent with peritoneal TB. She was managed with daily rifabutin ( $\mathrm{Rfb}), \mathrm{HZ}$ and ethambutol (E) together with daily pyridoxine. Her prednisolone dose was increased and tacrolimus levels monitored more frequently and doses adjusted accordingly. Her ALT did not rise beyond twice the ULN and she completed a standard six-month regimen of treatment. Mycobacterial culture confirmed a fully sensitive $M$. tuberculosis from both early morning urine and ascitic fluid samples. She made a full recovery and her graft continues to function well.
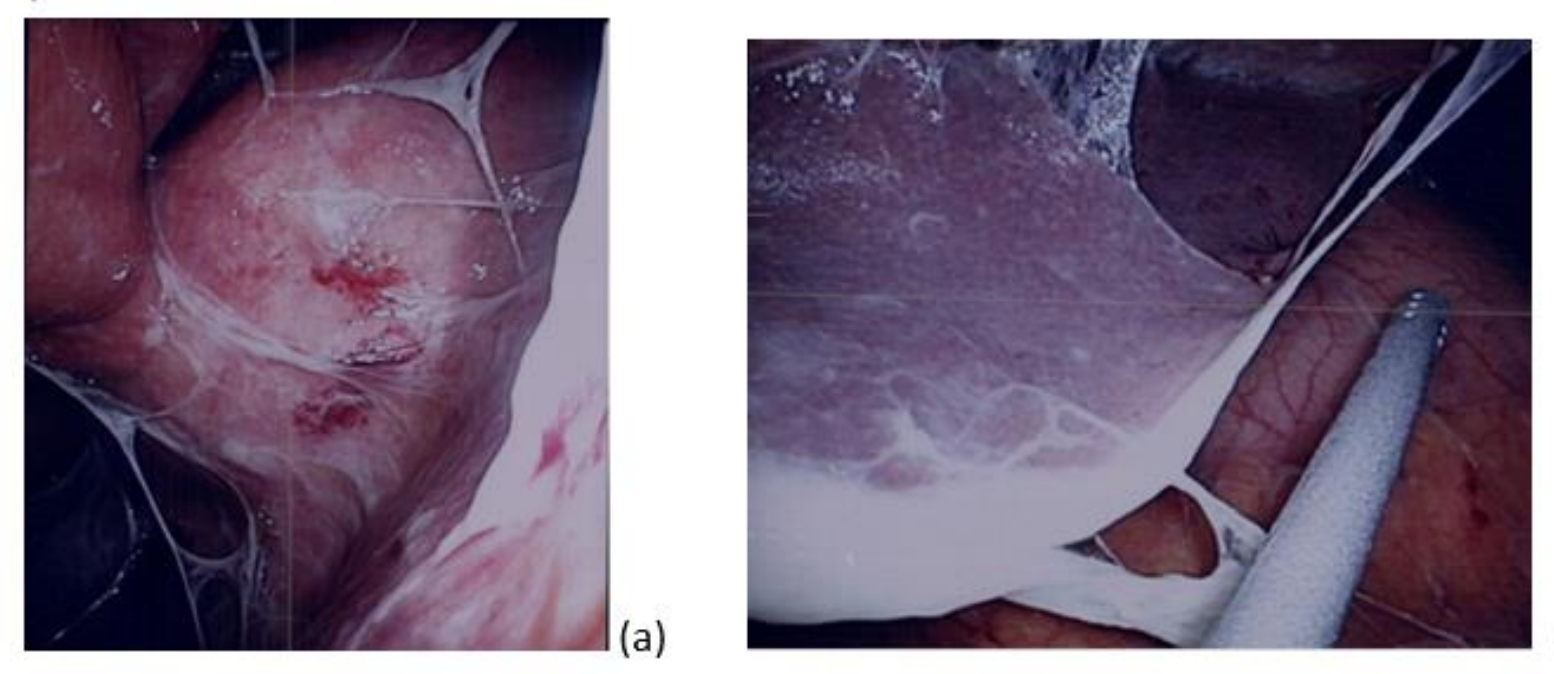

(b)

Figure 1 Laparoscopic view of peritoneum showing bowel adhered to caseous fibrinous strands in a renal transplant recipient with peritoneal TB.

\section{Case 3}

A 35 year-old male recipient of a simultaneous pancreas and kidney transplant for Type 1 diabetes mellitus (DM) presented with a four week history of cough, fever and breathlessness despite two courses of antibiotics from his General Practitioner. He was Caucasian, born in the UK and had not travelled beyond Western Europe. He had been transplanted 18 months previously and prior to receiving his transplant had been dialysis dependent for 12 months on CAPD. He had not 
received post-transplant chemoprophylaxis. Since then, his transplant had worked well and his immunosuppression was stable.

He denied weight loss or known TB exposure. His admission chest radiograph showed prominent hila as well as air-space shadowing in the right mid-zone (Figure 2). Routine culture of sputum, a respiratory viral swab, as well as tests for HIV, atypical pneumonia, EBV and cytomegalovirus were all non-contributory.

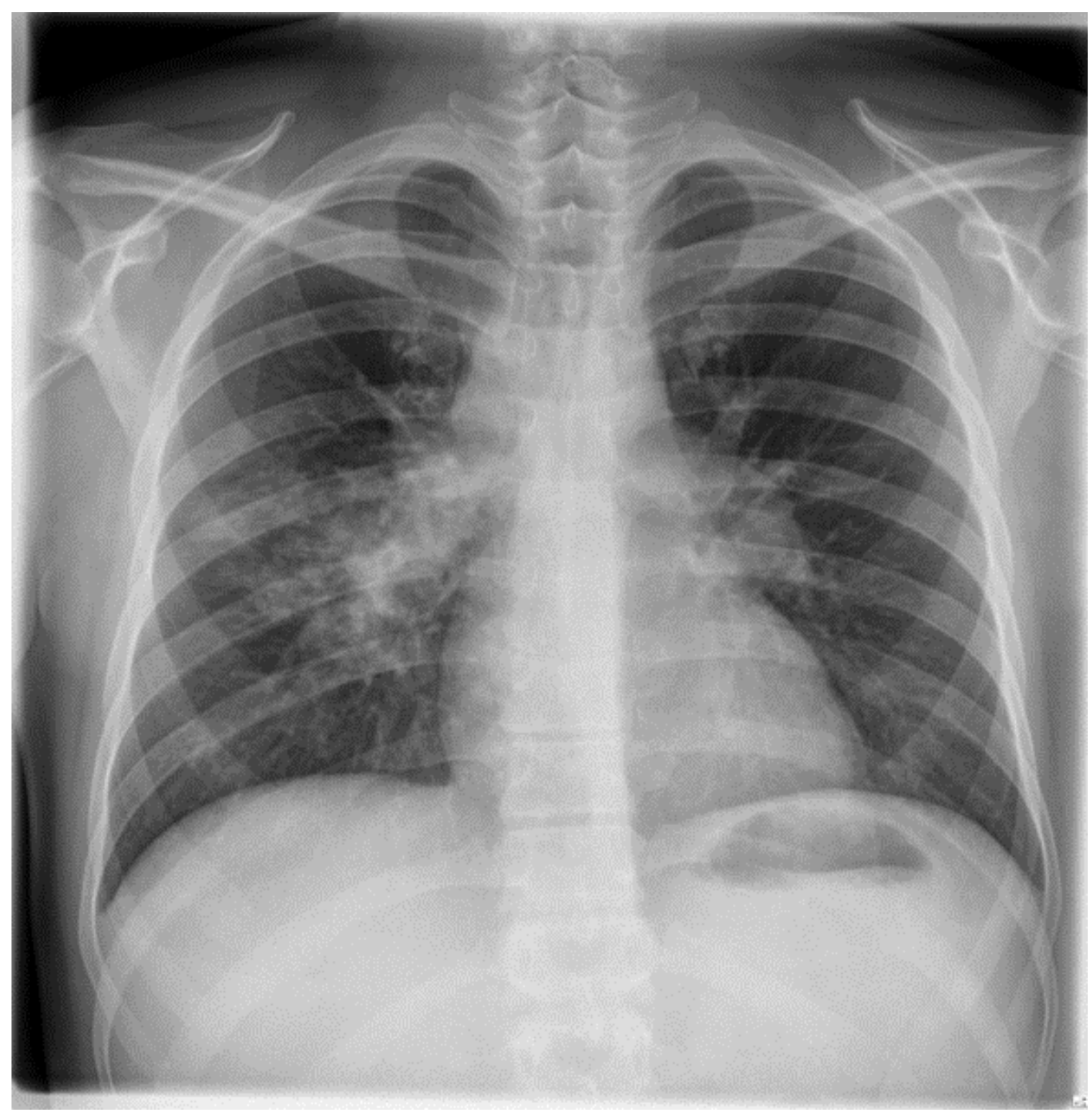

Figure $\mathbf{2}$ Chest radiograph showing prominent hilar with air-space opacities in the right mid-zone in a renal transplant recipient with pulmonary TB.

CT showed extensive asymmetrical mediastinal and bilateral hilar lymphadenopathy with extensive nodularity in the right lower lobe. The grafted pancreas and kidney enhanced normally. A post-transplant lympho-proliferative disorder was suspected. Bronchoscopy revealed multiple mucosal lesions at the entrance to the lower lobes. Biopsy showed evidence of patchy granulomatous infection with multiple AFBs seen. PCR confirmed M. tuberculosis with no evidence 
of rifampicin resistance and he was commenced on daily RfbHZE. On further review, no index case, or further epidemiological risk factors could be identified and he made an uncomplicated recovery.

\section{Case 4}

A 41 year-old female of Gambian origin presented with pain around a recently inserted right internal jugular (IJV) tunneled line for HD. She had developed CKD secondary to chronic tubulointerstitial nephritis with Sjogren's syndrome. Prior to her line insertion, a brachio-cephalic arteriovenous fistula had been created but had failed to mature and, despite a graft revision, had not functioned well.

She was febrile on admission and the skin overlying the tunneled line was erythematous and tender to touch. Her inflammatory markers were raised and she was managed for a suspected line infection. Cervical ultrasound showed thrombus within the right IJV and that the left IJV was patent. The right IJV line was removed and a temporary femoral line was secured for HD. She improved with antibiotic treatment and, prior to discharge, a new left IJV dialysis line was inserted.

One week later, she re-presented with persistent temperatures and tenderness, now over her cervical spine. An MRI excluded discitis and a trans-thoracic echocardiogram did not identify any vegetations. Blood cultures remained negative and she was managed with empirical antibiotics. CT showed an enlarged right sub-mandibular LN together with multiple small volume LN's in the neck, para-aortic and iliac chains. PET-CT showed intense FDG avidity within the right sub-mandibular LN and along multiple LN chains above and below the diaphragm as well as areas of high FDG uptake within the bowel and ileum. She continued to have temperatures up to $39^{\circ} \mathrm{C}$ and had lost $15 \mathrm{~kg}$ in weight since the onset of her symptoms. A lymphoproliferative disorder was suspected and core biopsies were arranged of the sub-mandibular LNs. Histology showed no evidence of lymphoma or carcinoma but did show non-necrotising granulomatous lymphadenitis. Stains for AFB and fungi were negative, as was immunocytochemistry for CMV and in situ hybridization (ISH) for EBV. Serum testing for human T-cell lymphotropic virus and human herpesvirus-8 were also negative as was an interferon gamma release assay (IGRA) for TB.

A presumptive diagnosis of TB lymphadenitis was made and, after a further biopsy was arranged for mycobacterial culture, she was started on a regimen appropriate to her weight of daily $\mathrm{RH}$ and pyridoxine together with EZ given three times weekly immediately after HD. She reported no known TB contacts and had not been treated for TB in the past. Tissue culture was negative for TB, but she responded well to anti-TB treatment and completed a six-month course.

\section{Discussion}

We describe four cases of TB disease in patients with advanced CKD, two of whom had wellfunctioning renal transplants on stable immunosuppression. Three patients were of Sub-Saharan heritage but all had been resident within the UK for over five years. These three patients also had predominantly extra-pulmonary sites of disease. In two cases, a lympho-proliferative disorder was thought to be the more likely diagnosis. Diagnosis in this patient group is not always straightforward, however a summary of how the authors apply a series of widely available diagnostic tests is included in Table 1. Mycobacterial confirmation is key and was possible in three cases, identifying fully sensitive organisms. All four patients recovered well. 
Table 1 Commonly used investigations for TB disease in advanced CKD.

\begin{tabular}{|c|c|}
\hline Investigations & Role \\
\hline CXR & $\begin{array}{l}\text { Role as part screening for those at risk of LTBI and as part of assessment for TB } \\
\text { disease. Typically, the presence of a calcified granuloma, apical pleural } \\
\text { thickening and pleural calcification may all indicate past TB exposure. }\end{array}$ \\
\hline Micro-biological & $\begin{array}{l}\text { An increased awareness of TB is important to ensure samples are sent for } \\
\text { mycobacterial culture along with bacterial and fungal culture. Repeat sampling } \\
\text { where practicable is advocated in those for whom samples are not initially sent } \\
\text { for TB culture. Early morning urine sent for TB culture is indicated in miliary and } \\
\text { renal TB. }\end{array}$ \\
\hline PCR & $\begin{array}{l}\text { Main role is in distinguishing between types of mycobacterial disease and } \\
\text { assessing for Rifampicin resistance on identification of } M \text {. tuberculosis. May } \\
\text { also have increased sensitivity than microscopy using ZN stains for AFB. }\end{array}$ \\
\hline ADA & $\begin{array}{l}\text { Included as part of fluid analysis of pleural effusions. In context of a lymphocytic } \\
\text { rich effusions, pleural ADA }>40 U / L \text { is suggestive of TB pleuritis [25]. Another } \\
\text { study based in a high incidence country found an ADA > or }=50 \mathrm{~L} / \mathrm{L} \text { was } 95 \% \\
\text { sensitive and } 89 \% \text { specific for TB [26]. }\end{array}$ \\
\hline IGRA & $\begin{array}{l}\text { Positive test can be associated with LTBI or active disease. Risk of false negative } \\
\text { in those who are immunosuppressed. }\end{array}$ \\
\hline TST & $\begin{array}{l}\text { Semi-quantitive role used to assess risk of LTBI. False positive can be associated } \\
\text { with BCG vaccination. Risk of a false negative in CKD as up to } 50 \% \text { of individuals } \\
\text { lack the cutaneous response. }\end{array}$ \\
\hline CT & $\begin{array}{l}\text { Role in assessment of unexplained weight loss, night sweats, pyrexia or } \\
\text { persistently raised inflammatory markers. Can guide further sampling for } \\
\text { histological and microbiological analysis. }\end{array}$ \\
\hline US & $\begin{array}{l}\text { Particular value in assessment of nodular pleural thickening but also to direct } \\
\text { image guided sampling of lymph nodes and pleural/abdominal effusions. }\end{array}$ \\
\hline FDG - PET & $\begin{array}{l}\text { Enhances value of CT to guide histological and microbiological sampling to sites } \\
\text { of active disease. Additionally can be useful to help exclude important } \\
\text { differential diagnoses including lymphoproliferative disorders and vasculitis. }\end{array}$ \\
\hline FOB +/- EBUS TBNA & $\begin{array}{l}\text { Fibreoptic bronchoscopy may be used in those with suspected endobronchial } \\
\text { disease or parenchymal abnormalities with use of bronchial washings. EBUS- } \\
\text { TBNA is used for sampling mediastinal and hilar lymph nodes. FOB may } \\
\text { additionally have a role in those with pleural disease. }\end{array}$ \\
\hline $\begin{array}{l}\text { Medical } \\
\text { thoracoscopy }\end{array}$ & Often considered in otherwise unexplained exudative pleural effusions \\
\hline
\end{tabular}

CXR; chest-xray, PCR; polymerase chain reaction, ADA; adenosine deaminase, IGRA; interferon gamma release assay, TST; tuberculin skin test, $\mathrm{CT}$; computed tomography, US; ultrasound, FDGPET; flurodeoxyglucose positron emission tomography, FOB; flexible bronchoscopy, EBUS-TBNA; endobronchial ultrasound transbronchial nodal aspiration. 


\section{Diagnostic Evaluation}

The majority of cases of TB disease in advanced CKD develop following reactivation of LTBI rather than a de novo exposure. Hence, a focused history around epidemiological risks or details of previously treated TB are vitally important in evaluating patients with unexplained systemic symptoms and/or abnormal imaging that could be consistent with TB disease. We have included a simple flow diagram to assist clinicians in the screening of LTBI and when to consider chemoprophylaxis (Figure 3). It is not possible to include all eventualities, but if in doubt, clinicians should seek advice from colleagues familiar with TB, usually a respiratory or infectious diseases physician.

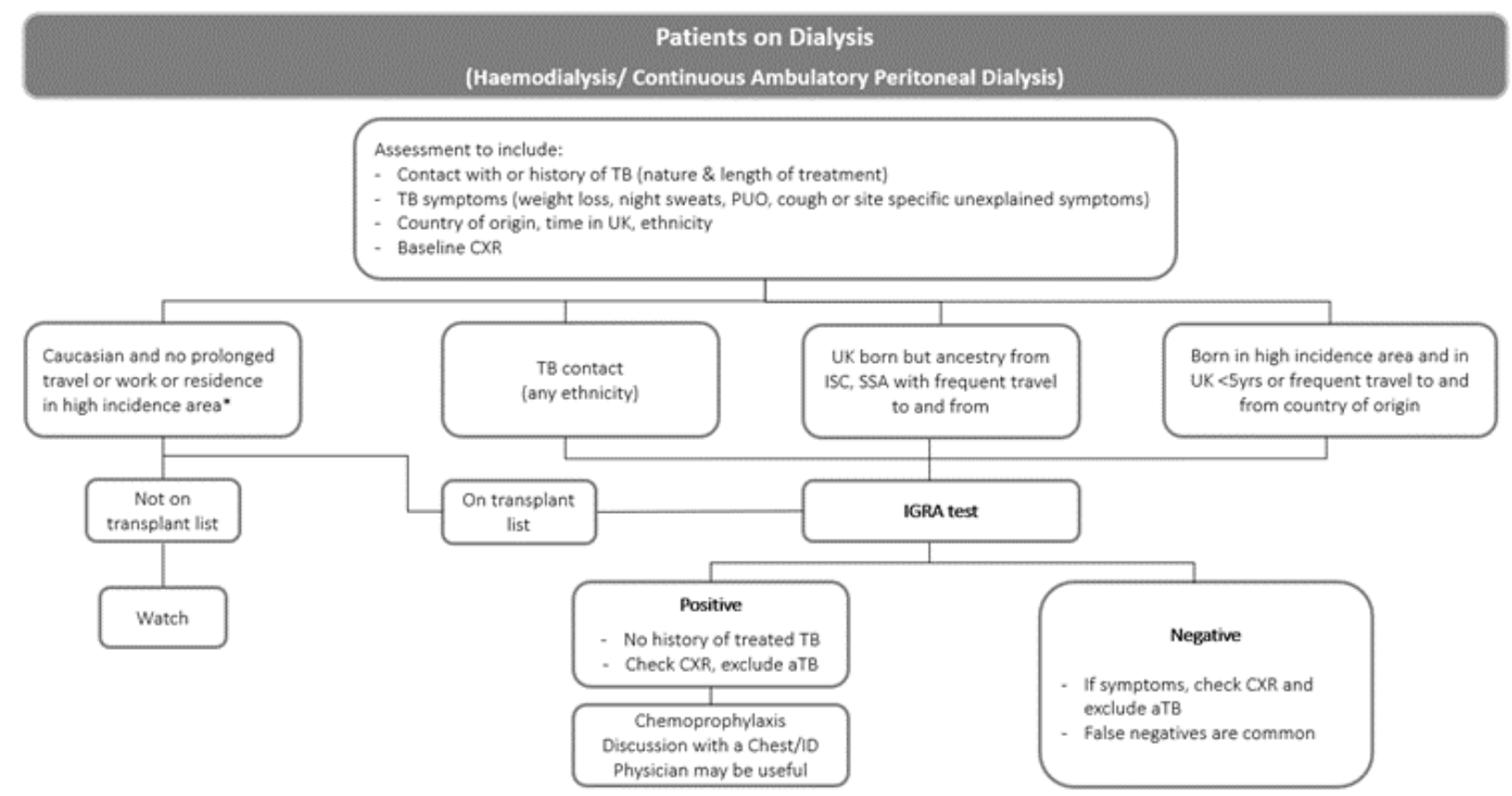

Figure 3 Flow diagram on the assessment of latent TB infection in patients on dialysis including those on transplant list. * WHO defines high incidence areas as $>40 / 100000$ cases and includes SSA, ISC, China, Eastern Europe and parts of South America In any patient developing a PUO, always consider TB in the differential diagnosis and search for active disease. Only use chemoprophylaxis if active TB excluded. If in doubt, consult a Chest or Infectious Diseases Physician specialising in TB. Abbreviations: ISC Indian sub-Continent; SSA sub-Saharan Africa; PUO pyrexia of unknown origin; pa per annum; CXR chest radiograph; aTB active tuberculosis; IgRA interferon gamma release assay.

\section{Choice of Anti-TB Medications and Dosing Regimens}

All four cases benefited from rifamycin and isoniazid containing regimens. In transplant recipients on immunosuppressive regimens as well as those with HIV co-infection on protease inhibitors, rifabutin is generally preferred as it is a less potent inducer of the CYP3A4 pathway than rifampicin $[12,13]$.

Three of the four cases described had a history of CAPD use. Although studies are few and relative risk difficult to determine, case reports support a high rate of peritoneal TB amongst 
patients who use CAPD $[14,15]$. The evidence base for managing TB infection in CAPD is limited. A single study proposed that no dose adjustment is needed for isoniazid, rifampicin or pyrazinamide [16]. TDM is, however, recommended in CAPD as demonstrated by the necessary dose adjustments in Case 1. Pre-dose trough levels are measured to ensure adequate drug clearance and thus limit the risk of toxicity, whilst post-dose levels taken at 2 and 4 hours can help to clarify that drug concentration levels are within the therapeutic target range to treat TB disease. A low post-dose level may indicate poor adherence to therapy or that the blood was taken at an inappropriate time relative to the last dose, so it is important these are considered before a dose adjustment is made. Table 2 lists the recommended therapeutic drug concentration levels for RHZE that we use at our centre in managing patients with TB disease on CAPD.

Table 2 Target therapeutic drug levels of first-line drugs in peritoneal dialysis.

\begin{tabular}{|c|c|c|c|c|}
\hline & Dosing regimen & $\begin{array}{l}\text { Trough }^{\#} \\
\text { (mg/L) }\end{array}$ & $\begin{array}{l}\text { Post dose* } \\
\text { (mg/L) }\end{array}$ & Comments \\
\hline Isoniazid & 300mg daily & $3-5$ & At $4 \mathrm{hrs}>10$ & $\begin{array}{l}\text { Is dialysed, clearance } \\
\text { mainly hepatobiliary }\end{array}$ \\
\hline Rifampicin & $\begin{array}{l}<50 \mathrm{~kg}: \quad 450 \mathrm{mg} \\
\text { daily } \\
\geq 50 \mathrm{~kg}: \quad 600 \mathrm{mg} \\
\text { daily }\end{array}$ & $<0.5$ & $\begin{array}{l}\text { At } 4 \text { hrs: } \\
<4 \text { sub-therapeutic } \\
4-8 \text { usually adequate } \\
8-24 \text { ideal }\end{array}$ & $\begin{array}{l}\text { Not dialysed, } \\
\text { clearance mainly } \\
\text { hepatobiliary }\end{array}$ \\
\hline Pyrazinamide & $\begin{array}{l}25-30 \mathrm{mg} / \mathrm{kg} \\
3 x / \text { week }\end{array}$ & $20-40$ & & Not dialysed \\
\hline Ethambutol & $\begin{array}{l}15-25 \mathrm{mg} / \mathrm{kg} \\
3 x / \text { week }\end{array}$ & $<1.0$ & $2-6$ & Not dialysed \\
\hline
\end{tabular}

"Adults. Always include pyridoxine. "Trough levels should be taken 24 hours post - dose.

* Where available.

Adapted from BTS 2010 guidelines on management of TB in CKD ${ }^{6}$.

Either ethambutol or moxifloxacin may be used as the fourth drug as part of first-line therapy in CKD. Ethambutol is normally excreted unchanged by the kidneys but can accumulate and cause dose-dependent ocular toxicity in renal failure [17]. It has improved efficacy when administered in normal doses less often than in a reduced daily dose [18] and is recommended at between $15-25$ $\mathrm{mg} / \mathrm{kg}$ three times/week in those with CKD stages 4 and 5, including those on HD [19]. Amongst transplant recipients with maintained graft function, as in Cases 2 and 3, the recommended dose is $15 \mathrm{mg} / \mathrm{kg}$ given daily. In view of the challenges of completing TDM in the clinic, our local policy is to manage patients on CAPD and HD with moxifloxacin $400 \mathrm{mg}$ daily as an alternative to ethambutol.

\section{Lessons Learnt}


In our case series, Case 2 was given chemoprophylaxis post-transplant but this was withdrawn after only eight days following a four-fold rise in her serum ALT. BTS guidance is to stop isoniazid if the ALT/AST level rises to five times the ULN or if the bilirubin level rises, allow levels to normalise, then re-introduce $\mathrm{H}$ at $50 \mathrm{mg} /$ day, increasing sequentially by $50 \mathrm{mg}$ over several days thereafter to the target dose [20]. Best practice would have been to either re-introduce low dose isoniazid, or alternatively, to use rifampicin $10 \mathrm{mg} / \mathrm{kg}$ for $4-6$ months. Arguably, this may have prevented her subsequent infection.

Case 3 was a Caucasian male, who had no known TB exposure or risk beyond that of being on immunosuppressive medication and having CKD and diabetes. It is possible that a screening programme including routine use of IGRA testing would have identified him as at risk of LTBI.

Cases 1 and 4, were dialysis dependent using CAPD and HD respectively and had not been identified as at high risk of TB infection and screened for LTBI. Both patients were, however, of subSaharan African origin and a focused history of their risk of TB infection would have revealed epidemiological risk factors and, in Case 1, prior contact and symptoms of active disease.

In 3 of the 4 cases, a history of contact and a completed course of TB chemoprophylaxis would have contributed to possible prevention of subsequent TB disease. In addition, an IGRA test could have added evidence of LTBI in Case 3 who had no known epidemiological risks. This would have enabled chemoprophylaxis before transplant after first ensuring that active disease was not present. Although tissue samples and IGRA testing were negative in Case 4, the patient originated from subSaharan Africa, had a pyrexia and weight loss, other possible diagnoses had been excluded and she responded well to treatment for TB. It is not unusual to have difficulty confirming a diagnosis of active TB in CKD and following transplantation due to the mixed underlying immunodeficiency as discussed above. This case, however, underlines the importance of remaining aware of TB as a potential cause of fever and weight loss, especially in patients of African and Asian origin.

\section{Role of Screening in Preventing TB Infection in CKD Stages 4 and 5}

The increased risk of TB in CKD is at least in part related to immunodeficiency which is multifactorial. Changes in immunity begin as early as stage 3 CKD and deteriorate in later stages as kidney function worsens and waste products accumulate [21]. Advanced CKD is associated with disturbances of both the innate and adaptive immune systems. Pro-inflammatory cytokines accumulate in part as a consequence of decreased renal elimination and there is increased generation of uraemic toxins and oxidative stress [21]. Within the adaptive immune system, a variety of immune related cells demonstrate impaired function, not least evidenced by the reduced effectiveness of the tuberculin skin test to diagnose LTBI in many patients.

Since the BTS Guidelines were published in 2010, the widespread use of chemoprophylaxis (usually with six months of isoniazid with pyridoxine) in high-risk transplant recipients has, in all likelihood, helped reduce the rate of TB infection and disease within this cohort of patients. An audit completed at our own centre, found that out of 912 patients who underwent renal transplantation between 2011 - 2014, 88\% correctly received isoniazid prophylaxis according to Hospital guidelines and, during this time, only one patient developed TB post-transplant (Case 2) [22]. However, the evidence base for the use of chemoprophylaxis in solid organ transplantation is limited and a Cochrane review [23] on this subject identified only three eligible randomised control studies that were suitable for inclusion. Amongst a total of 558 kidney transplant recipients randomised to 
receive either anti-TB prophylaxis (isoniazid) or no chemoprophylaxis, participants who received anti-TB prophylaxis were $65 \%$ less likely to develop TB: RR $0.35,95 \% \mathrm{Cl} 0.14$ to 0.89 . These studies were however based in areas with high TB incidence (India and Pakistan) and therefore may be less applicable in areas where TB prevalence is low. The studies also recorded high rates of liver dysfunction among those who received anti-TB prophylaxis although this seems likely to have been related to high rates of co-infection with hepatitis $B$ and $C$ viruses.

The role of TB screening programmes in patients with advanced stage CKD remains controversial. Historically, the case against screening all patients on renal transplant lists has been that not all will proceed to transplantation and be exposed to immunosuppressive medication. Such an approach, however, neglects the increased RR of TB infection and disease associated with HD and CAPD. While some evidence was based on expert opinion at the time, the 2010 BTS Guidelines advocate that all patients with CKD at risk of TB should be screened with a focused history, clinical examination, a chest radiograph and if appropriate an IGRA test (Figure 3). Since the publication of these guidelines, there have been further publications on the use of screening in this population [24]. Indeed, a recently published account of our own experience between 1994-2010 found that, compared with background London TB rates, the risk of developing TB disease was significantly higher [7], further supporting the case for screening appropriate patients on RRT.

The widespread use of chemoprophylaxis in high-risk individuals undergoing renal transplantation has been successful in reducing rates of TB infection post-transplant. With accumulating evidence for increased risks in patients on RRT, however, it is now time to consider extending those benefits to patients with advanced CKD through effective and dedicated screening programmes.

\section{Abbreviations}

$\begin{array}{ll}\text { Abbreviation } & \text { Meaning } \\ \text { AFB } & \text { Acid fast bacilli } \\ \text { CAPD } & \text { Continuous ambulatory peritoneal dialysis } \\ \text { CKD } & \text { Chronic kidney disease } \\ \text { CT } & \text { Computed tomogram } \\ \text { E } & \text { Ethambutol } \\ \text { EBV } & \text { Epstein-Barr virus } \\ \text { FSGS } & \text { Focal sclerosing glomerular sclerosis } \\ \text { H } & \text { Isoniazid } \\ \text { HIV } & \text { Human immunodeficiency virus } \\ \text { IGRA } & \text { Interferon gamma release assay } \\ \text { IJV } & \text { Internal jugular vein } \\ \text { LTBI } & \text { Latent Tuberculosis Infection } \\ \text { PCR } & \text { Polymerase chain reaction } \\ \text { R } & \text { Rifampicin } \\ \text { Rfb } & \text { Rifabutin } \\ \text { RR } & \text { Relative Risk } \\ \text { RRT } & \text { Renal replacement therapy }\end{array}$


TB Tuberculosis

Z Pyrazinamide

ZN Ziehl-Neelsen

\section{Acknowledgments}

The authors are grateful for additional information on drug monitoring levels provided by toxicology laboratories in Bristol and Cardiff, UK.

\section{Author Contributions}

SM, PC, RB and HM were all involved in the care of at least one of the cases described. GDA provided pharmacological advice. All authors contributed to the drafting and final approval of the article.

\section{Competing Interests}

None.

\section{References}

1. Hu HY, Wu CY, Huang N, Chou YJ, Chang YC, Chu D. Increased risk of tuberculosis in patients with end-stage renal disease: A population-based cohort study in Taiwan, a country of high incidence of end-stage renal disease. Epidemiol Infect. 2014; 142: 191-199.

2. Lightstone L. Preventing renal disease: The ethnic challenge in the United Kingdom. Kidney Int. 2003; 63: S135-S138.

3. Duarte R, Lönnroth K, Carvalho C, Lima F, Carvalho AC, Muñoz-Torrico M, et al. Tuberculosis, social determinants and co-morbidities (including HIV). Pulmonology. 2018; 24: 115-119.

4. Costa SD, de Sandes-Freitas TV, Jacinto CN, Martiniano LV, Amaral YS, Paes FJ, et al. Tuberculosis after kidney transplantation is associated with significantly impaired allograft function. Transpl Infect Dis. 2017; 19: e12750.

5. Singh N, Paterson DL. Mycobacterium tuberculosis infection in solid-organ transplant recipients: Impact and implications for management. Clin Infect Dis. 1998; 27: 1266-1277.

6. Milburn H, Ashman N, Davies P, Doffman S, Drobniewski F, Khoo S, et al. Guidelines for the prevention and management of Mycobacterium tuberculosis infection and disease in adult patients with chronic kidney disease. Thorax. 2010; 65: 559-570.

7. Ostermann M, Palchaudhuri P, Riding A, Begum P, Milburn HJ. Incidence of tuberculosis is high in chronic kidney disease patients in South East England and drug resistance common. Ren Fail. 2016; 38: 256-261.

8. Al-Efraij K, Mota L, Lunny C, Schachter M, Cook V, Johnston J. Risk of active tuberculosis in chronic kidney disease: A systematic review and meta-analysis. Int J Tuberc Lung Dis. 2015; 19: 1493-1499.

9. Muñoz L, Santin M. Prevention and management of tuberculosis in transplant recipients: From guidelines to clinical practice. Transplantation. 2016; 100: 1840-1852.

10. Naqvi A, Rizvi A, Hussain Z, Hafeez S, Hashmi A, Akhtar F, et al. Developing world perspective of posttransplant tuberculosis: Morbidity, mortality, and cost implications. Transplant Proc. 2001; 


$$
\text { 33: } 1787-1788 .
$$

11. Myall K, Milburn HJ. An update on the management of latent tuberculosis infection and active disease in patients with chronic kidney disease. Pol Arch Intern Med. 2017; 127: 681-686.

12. Grassi C, Peona V. Use of rifabutin in the treatment of pulmonary tuberculosis. Clin Infect Dis. 1996; 22: S50-S54.

13. Narita M, Stambaugh JJ, Hollender ES, Jones D, Pitchenik AE, Ashkin D. Use of rifabutin with protease inhibitors for human immunodeficiency virus-infected patients with tuberculosis. Clin Infect Dis. 2000; 30: 779-783.

14. Malik GH, Al-Harbi AS, Al-Mohaya S, Al-Khawajah H, Kechrid M, Al Hassan AO, et al. Eleven years of experience with dialysis associated tuberculosis. Clin Nephrol. 2002; 58: 356-362.

15. Quantrill SJ, Woodhead MA, Bell CE, Hutchison AJ, Gokal R. Peritoneal tuberculosis in patients receiving continuous ambulatory peritoneal dialysis. Nephrol Dial Transplant. 2001; 16: 1024 1027.

16. Ahn C, Oh KH, Kim K, Lee KY, Lee JG, Oh MD, et al. Effect of peritoneal dialysis on plasma and peritoneal fluid concentrations of isoniazid, pyrazinamide, and rifampin. Perit Dial Int. 2003; 23: 362-367.

17. Citron KM. Ethambutol: A review with special reference to ocular toxicity. Tubercle. 1969; 50: 32-36.

18. Ellard GA. Chemotherapy of tuberculosis for patients with renal impairment. Nephron. 1993; 64: 169-181.

19. Lundin AP, Adler AJ, Berlyne GM, Friedman EA. Tuberculosis in patients undergoing maintenance hemodialysis. Am J Med. 1979; 67: 597-602.

20. Joint Tuberculosis Committee of the British Thoracic Society. Chemotherapy and management of tuberculosis in the United Kingdom: Recommendations 1998. Thorax. 1998; 53: 536-548.

21. Romanowski K, Clark EG, Levin A, Cook VJ, Johnston JC. Tuberculosis and chronic kidney disease: An emerging global syndemic. Kidney Int. 2016; 90: 34-40.

22. Periselneris JN, Mahendran S, Chowdhury P, Milburn H. P110 the role of TB chemoprophylaxis in renal transplant recipients. Thorax. 2016; 71: A142-A143.

23. Adamu B, Abdu A, Abba AA, Borodo MM, Tleyjeh IM. Antibiotic prophylaxis for preventing post solid organ transplant tuberculosis. Cochrane Database Syst Rev. 2014; 3: CD008597.

24. Sester M, Van Leth F, Bruchfeld J, Bumbacea D, Cirillo DM, Dilektasli AG, et al. Risk assessment of tuberculosis in immunocompromised patients. A TBNET study. Am J Respir Crit Care Med. 2014; 190: 1168-1176.

25. Light RW. Update on tuberculous pleural effusion. Respirology. 2010; 15: 451-458.

26. Diacon AH, Van de Wal BW, Wyser C, Smedema JP, Bezuidenhout J, Bolliger CT, et al. Diagnostic tools in tuberculous pleurisy: A direct comparative study. Eur Respir J. 2003; 22: 589-591. 


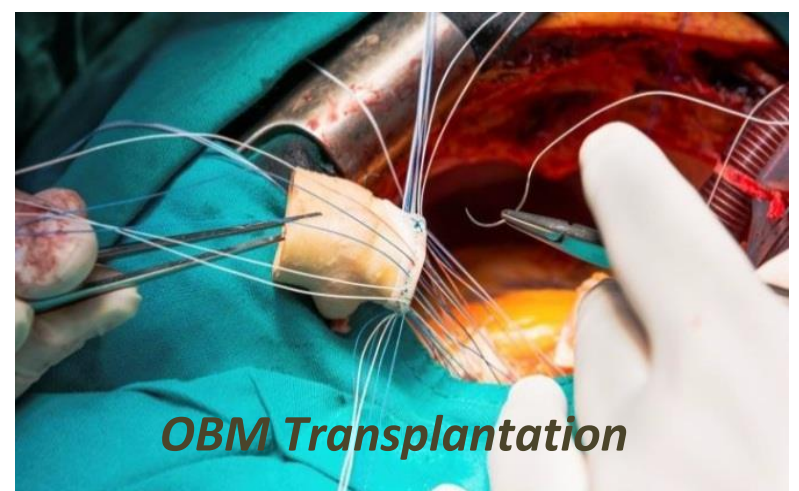

Enjoy OBM Transplantation by:

1. Submitting a manuscript

2. Joining in volunteer reviewer bank

3. Joining Editorial Board

4. Guest editing a special issue

For more details, please visit:

http://www.lidsen.com/journals/transplantation 